\title{
The QUEST for better marks
}

\author{
Edward D. Matsumoto, MD, MEd, FRCSC
}

Faculty of Health Sciences, Department of Surgery, Division of Urology, McMaster University, Hamilton, ON, Canada

Cite as: Can Urol Assoc J 2019;13(2):43-4. http://dx.doi.org/10.5489/cuaj.5830

See related article on page 39

T he paper by Touma and colleagues in this issue of CUAJ represents a critical element of urology residency training - that of preparing residents for successful completion of the Royal College of Physicians and Surgeons of Canada (RCPSC) certification examination that culminates the five years trainees have progressed through residency, further preparing them for fellowships and ultimately independent practice. Touma et al present data on the first 20 years (1997-2016) of the Queen's Urology Examination Skills Training (QUEST) program, describing the impact this national preparatory exam has had on the performance of graduating urology residents. ${ }^{1}$ This work is incredibly timely and valuable, as the RCPSC's Competence by Design (CBD) ${ }^{2}$ curriculum initiative has begun its implementation into all postgraduate medical training throughout Canada. The current, traditional, time-based structured urology residency curriculum components, such as didactic lectures, surgical foundations, journal clubs, rounds, participation in scholarly research, simulation sessions, rotation experience, and intraining exams all aim to prepare residents to meet the core CanMEDS competencies. However, with the implementation of CBD, we will see changes to urology curricula, alongside redefined goals and objectives. Via a retrospective analysis of 495 urology candidates' performance over the first 20 years of QUEST, Touma and colleagues have importantly assessed the current state of knowledge regarding Canadian urology residency programs conducted under the traditional time-based training model, providing potentially advantageous curricula development guidance as CBD is further developed and integrated into Canadian urology programs.

QUEST's aim is to simulate the RCPSC's certification examination by providing graduating residents with an exam comprised of a written component of short answer questions (SAQs) and an oral component via an eight-station OSCE. Without a doubt, QUEST has been an instrumental tool for both Canadian urology residents and program directors to gauge preparedness for the high-stakes RCPSC examination.
In 2004, Dr. Andrew MacNeily and colleagues showed that during the first five years of QUEST, residents' performance on QUEST and the RCPSC certification exam was moderately and significantly correlated $(r=0.68 ; p<0.001)$, and likewise with QUEST and the American Board of Urology part 1 qualifying exam (ABU 1) $(r=0.42 ; p=0.005)$. This demonstrated that QUEST has been a moderate predictor of performance on these exams. ${ }^{3}$ Program directors and final-year urology residents use this well-known fact to fine-tune studying for the remaining months leading up to the final RCPSC exam. A recent study by Skinner and colleagues reported that $73 \%$ of Canadian urology residents spend more than 25 hours per week studying in their final year, with $99 \%$ reporting that the RCPSC exam was the biggest motivator for studying in their final year. ${ }^{4}$ Throughout the QUEST program, residents receive feedback on areas requiring further attention; the SAQ and OSCE feedback has been an important objective of the QUEST examination and undoubtedly plays a role in the direction residents will take during their final months of studying for the RCPSC exam. The QUEST program has been a vital part of Canadian urological training and has become part of the "rite of passage" for chief urology residents.

Based on the QUEST data, Dr. Touma and associates' study clearly show that the overall marks were found "to fit within one standard deviation from the respective means for all 12 training programs." ${ }^{1}$ This is indicative of the fact that all Canadian urology residency programs are, in fact, preparing their residents well for the RCPSC certification exam, regardless of the lack of a standardized and uniform urology residency curriculum in Canada. Despite differences among urology programs' size, geography, and resources, residents in their chief year across Canada are performing with minimal variance on both the written and OSCE portions of the QUEST exam.

However, the authors do show, with certain consistency, that several programs appear to prepare residents better for both the written exams and OSCEs at QUEST, while some programs appear to consistently underprepare their residents for the QUEST exam. I do not advocate revealing the identities of the programs, but as a future study, Canadian urology educators would likely appreciate seeing the characteristics of programs training residents who consistently 
perform well at QUEST. Certainly, many variables may play into the preparedness of residents to take these exams, as were outlined in the paper: the changes in programs and faculty turnover; residents may gravitate to programs that best suit their needs and objectives (e.g., admission of international medical graduates); and research productivity, among others. Furthermore, as the authors explained, due to the nature of the retrospective review and the data itself, residents' surgical and clinical skills and various characteristics of the CanMEDS roles, among other factors that can influence RCPSC exam preparedness, are not described in this study. It is difficult to say which factors are truly indicative of this performance; perhaps some residents are taught to be or are just innately better test-takers; or it could be due to meaningful teaching interventions and unique characteristics that are incorporated into each residency curriculum. I look forward to seeing a prospective study that identifies the factors that contribute to resident preparedness for multiple aspects, beyond the written exams and OSCEs.

As surgical educators, we can take pride and confidence in knowing that residents graduating from Canadian urology programs are competent and have the knowledge to secure fellowships and be successful, independent urologists. This is a reflection on the time and energy spent by program directors, faculty members, program assistants, and the universities across Canada. Furthermore, the Chair of the Urology Subspecialities Committee, led by the likes of Dr. Andrew MacNeily and incoming Chair, Dr. Keith Rourke, along with the program directors, have shown a unified and outstanding cooperation with regards to improving urology residency training in Canada. I can personally attest to the dedication of this group through the recent development and implementation of the CBD program in urology. What remains to be seen in the future is whether there will be less of a reliance on high-stakes examination as we continue with the CBD integration. If this is the case, then the role of QUEST may have to evolve as well. I commend the authors on this important and very timely study, and I look forward to their future research.

Competing interests: The author reports no competing personal or financial interest related to this work.

\section{References}

1. Touma NJ, Beiko DT, MacNeily AE, et al. Impact of a training program on the performance of graduating Canadian residents on a national urology exam: Results of the last 20 years. Can Urol Assoc J 2018 July 31; Epub ahead of print. ttp://dx.doi.org/10.5289/cuaj.5386

2. Competence by Design: ebook. Available at: www.royalcollege.ca. Accessed Dec. 17, 2018.

3. MacNeily AE, Baverstock RJ, Cole G, et al. Quantitative assessment of a new preparatory tool for board certification in urology. BJU Int 2004;93:558-61. https://doi.org/10.1111/i.1464-410X.2003.04661.x

4. Skinner TAA, Ho L, Touma NJ. Study habits of Canadian urology residents: Implications for development of a competence by design curriculum. Can Urol Assoc J 2017;11:83-7. https://doi.org/10.5489/cuaj.4132

Correspondence: Dr. Edward D. Matsumoto, Faculty of Health Sciences, Department of Surgery, Division of Urology, McMaster University, Hamilton, 0N, Canada; matsumo@mcmaster.ca 